\title{
Effects and mechanisms of CTRP3 overexpression in secondary brain injury following intracerebral hemorrhage in rats
}

\author{
YU WAN $^{1,2}$, JIEQIONG WANG $^{1,2}$, BO YANG $^{1,2}$, CONGGAI HUANG $^{1,2}$, \\ XIAOQIN TANG ${ }^{1,2}$, HONG YI ${ }^{1,2}$, YUN LIU $^{1,2}$ and SHAOHUA WANG ${ }^{1,2}$ \\ ${ }^{1}$ Department of Pathology, ${ }^{2}$ Medical Experiment Research Center, The Affiliated Hospital \\ of Southwest Medical University, Luzhou, Sichuang 646000, P.R. China
}

Received July 23, 2021; Accepted October 15, 2021

DOI: $10.3892 /$ etm.2021.10957

\begin{abstract}
C1q/TNF-related protein-3 (CTRP3) is a novel adipokine that serves an important role in oxidative stress, anti-apoptosis, anti-inflammation and immune regulation. The aim of the present study was to investigate the protective role of CTRP3 against intracerebral hemorrhage ( $\mathrm{ICH})$-induced brain injury. A model of autologous arterial blood-induced ICH was constructed in rats. Intracerebral infusion of a lentivirus carrying the CTRP3 gene was used to induce CTRP3 overexpression in the brain. The effects and mechanisms of CTRP3 overexpression on brain injury were investigated by detecting brain edema, blood-brain barrier (BBB) integrity, neurological function and inflammatory-associated factors 3 days after $\mathrm{ICH}$. The present results demonstrated that CTRP3 overexpression ameliorated $\mathrm{ICH}$-induced neurological dysfunction, decreased brain edema, maintained BBB integrity and attenuated inflammation. The protective effect of CTRP3 overexpression was associated with increased activation of silent information regulator 1 (SIRT1). In conclusion, the present study demonstrated that CTRP3 overexpression protected against ICH-induced brain injury in rats, potentially via activating the SIRT1 signaling pathway.
\end{abstract}

\section{Introduction}

Intracerebral hemorrhage ( $\mathrm{ICH})$ is the most devastating subtype of stroke and exhibits a poor functional prognosis and high mortality rate worldwide (1-3). Clinically, patients with ICH, except those with severe symptoms and surgical indications (Bleeding volume $>30 \mathrm{ml}$; Glasgow Coma index score $\geq 5$ ), are usually administered conservative medical treatments (4). Secondary brain injury (SBI) following ICH

Correspondence to: Professor Shaohua Wang, Department of Pathology, The Affiliated Hospital of Southwest Medical University, 25 Taiping Street, Luzhou, Sichuang 646000, P.R. China

E-mail: wangshaohua1005@swmd.edu.cn

Key words: C1q/TNF-related protein-3, intracerebral hemorrhage, brain edema, blood-brain barrier, neurological dysfunction serves an important role in the deterioration of neurological function $(5,6)$. The mechanisms underlying ICH-induced SBI are complex and involve many factors, such as destruction of the blood-brain barrier (BBB), release of thrombin, toxicity from erythrocyte lysis and its products, inflammatory reactions, immune-mediated damage and excessive production of reactive oxygen species that are involved in complement system (7). The inflammatory response induced by $\mathrm{ICH}$ serves a key role in the continuous development of SBI. For example, blood components leaking into the brain lead to activation of immune cells, such as microglia. This leads to the destruction of the BBB and infiltration of peripheral blood leukocytes into the brain tissue, resulting in the production of a large number of inflammatory factors that induce brain edema, ultimately leading to dysfunction and death of both neurons and glia (8-11). Therefore, interventions that regulate $\mathrm{ICH}$-induced inflammatory responses are important for the treatment of ICH-induced brain injury. Silent information regulator 1 (SIRT1), an NAD ${ }^{+}$-dependent protein deacetylase, is involved in a variety of disease processes, such as inflammation, cell death and metabolism, by regulating targets via deacetylation (12). Evidence has shown that SIRT1 exerts a neuroprotective effect following ICH (13). SIRT1 protects against ICH-induced brain damage via inhibiting neuroinflammation by deacetylating $\mathrm{NF}-\kappa \mathrm{B} / \mathrm{p} 65$ (14).

C1q/TNF-related proteins (CTRPs) are a newly discovered and highly conserved family of adiponectin paralogs, which comprises $\geq 15$ members (CTRP1-15) (15). CTRPs are widely distributed and are involved in regulating many physiological or pathological processes, such as substance metabolism, vasodilation and inflammatory reactions. CTRP3 is a member of this family that is expressed by adipocytes, adipose stromal cells and other types of cell and exhibits homology with the genomic structure of adiponectin $(16,17)$. Furthermore, CTRP3 serves an important role in inflammation, metabolism, anti-apoptosis, angiogenesis and cardioprotection (18). To the best of our knowledge, however, at present, there is a lack of research regarding the effects of CTRP3 on ICH. Thus, the aim of the present study was to investigate the protective effects and mechanisms of CTRP3 in a rat model of ICH. 


\section{Materials and methods}

Animals. Adult male Sprague-Dawley (SD) rats $(\mathrm{n}=104$; weight, $240 \pm 20 \mathrm{~g}$; age, $12.5 \pm 0.1$ weeks) were purchased from the Experimental Animal Center of Southwest Medical University (Luzhou, China). All animal studies were approved by the Biomedical Ethics Committee of Southwest Medical University (approval no. 20210223-135). All experimental procedures were in accordance with guidelines for the care and use of laboratory animals of the National Institutes of Health (19). Rats were placed in standard cages and exposed to a $12 / 12$-h light/dark cycle at a constant room temperature of $24-26^{\circ} \mathrm{C}$ and $60 \%$ indoor relative humidity and were provided food and water ad libitum. For euthanasia, rats were anesthetized by an intraperitoneal injection of 3\% pentobarbital sodium ( $30 \mathrm{mg} / \mathrm{kg}$ body weight). Once fully anesthetized, rats were sacrificed by cervical dislocation and death was confirmed by cessation of breathing and faded eye color.

Experimental design. To investigate the effects of CTRP3 in an ICH model established via autologous blood injection, rats were randomly distributed into four groups: i) Sham surgery (Sham, $n=33)$; ii) ICH ( $\mathrm{n}=31 ; 3$ died); iii) ICH + null-vector control (Lenti.Null, $\mathrm{n}=33$; 5 died) and iv) $\mathrm{ICH}+$ lentiviral CTRP3-overexpression (Lenti.CTRP3, $\mathrm{n}=34 ; 5$ died). Behavioral tests, measurement of brain water content, evaluation of BBB permeability, western blotting and ELISA were performed 3 days following ICH.

To determine whether the SIRT1 signaling pathway was involved in CTRP3-induced neuroprotection, rats were randomized to the following groups: $\mathrm{ICH}+$ Lenti. Null ( $\mathrm{n}=8 ; 1$ died), ICH + Lenti.CTRP3 ( $\mathrm{n}=22 ; 2$ died), ICH + Lenti.CTRP3 + EX527 (SIRT1 inhibitor, $n=22 ; 2$ died) and $\mathrm{ICH}+$ vehicle $(\mathrm{n}=21 ; 1$ died). Lenti-CTRP3 was injected intraventricularly 14 days before ICH. EX527 was injected intraventricularly $30 \mathrm{~min}$ before ICH. Modified Garcia test, BBB integrity/permeability, brain edema and IL-1 $\beta$, and TNF- $\alpha$ levels were assessed 3 days after ICH.

ICH rat model. A rat model of striatal ICH was established by injecting autologous blood into the rat right striatum, as previously described (20). SD rats were anesthetized by intraperitoneal injection of pentobarbital sodium $(66 \mathrm{mg} / \mathrm{kg})(21)$. Then, rats were horizontally fixed on the platform of a stereotaxic frame (David Kopf Instruments) in the prone position. Autologous blood (50 $\mu \mathrm{l})$, which was drawn from the femoral artery, was injected into the striatum $(0.2 \mathrm{~mm}$ anterior, $3.0 \mathrm{~mm}$ laterally to the right of bregma and $5.8 \mathrm{~mm}$ deep from the surface of the skull) via a microsyringe pump. Sham rats were injected with an equal volume of saline. After the operation, each rat was placed into a separate cage and body temperature maintained at $37.0 \pm 0.5^{\circ} \mathrm{C}$ before follow-up experiments were conducted.

Injection of lentiviral CTRP3 gene and administration of EX527. Lentivirus harboring CTRP3 and empty vector were constructed by Shanghai GeneChem Co., Ltd. with green fluorescent protein. Rats were administered $5 \mu \mathrm{l}$ CTRP3 lentivirus $\left(1 \times 10^{9}\right.$ titer units/ml, diluted 10X with Enhanced Infection Solution). Enhanced Infection Solution (cat. no. REVG002A;
Shanghai GeneChem Co., Ltd.) was used to improve the efficiency of virus infection. The lentivirus CTRP3 gene was injected into the right ventricle $(0.9 \mathrm{~mm}$ lateral to the sagittal suture, $1.9 \mathrm{~mm}$ posterior to the coronal suture and $3.5 \mathrm{~mm}$ deep into the cortex) 14 days before ICH, as previously reported (20). The Lenti.Null rats were subjected to the same procedure. Then, EX527, a SIRT1 inhibitor (Selleck Chemicals) or vehicle was also injected intraventricularly $1 \mathrm{~h}$ before induction of ICH. The dose of EX527 $(10 \mu \mathrm{g} / 5 \mu \mathrm{l}$ dissolved in 10\% DMSO) was selected as previously described (22). The needle was withdrawn after 15 min injection. Each rat was then placed back into an individual cage and provided food and water ad libitum.

Behavioral tests. Neurological function was evaluated by the Garcia, beam walking and wire hanging test, as previously described (20). The Garcia test consists of six tests, including autonomous activity, limb activity symmetry, table-edge forepaw extension, grab cage and sensory touch response tests. Each test yields a score of $0-3$; the minimum overall score was 0 and the maximum overall score was 18 . Lower scores are indicative of more serious neuronal damage. In the beam walking test (23), a square wood bar $(590 \times 25 \mathrm{~mm})$ was placed $100 \mathrm{~mm}$ from the ground. Each rat was allowed to walk on the balance beam. The rats were assessed to determine if they could use their limbs symmetrically to reach the platform at the end of the beam and given a score of $0-5$ as follows: 0 , fell from the beam; 1 , sat on the beam but did not walk; 2 , jumped onto the beam but did not move forward; 3 , jumped onto the beam and walked with contralateral hind limbs of lesion slipping down the beam $>50 \%$ of the time; 4 , jumped onto the beam and walked with contralateral hind limbs slipping down the beam $<50 \%$ of the time and 5, jumped onto the beam and walked without falling. Each rat's performance was calculated as the mean score of three trials. In the wire hanging test (24), the rats were placed in the center of a $600 \times 2 \mathrm{~mm}$ wire $50 \mathrm{~cm}$ from the ground with a sponge pad underneath. Each rat was observed four times for $30 \mathrm{sec}$ each. The rats were scored based on the time they were suspended from the wire and the position of the limbs as follows: 0 , fell $<30 \mathrm{sec}$; 1 , did not fall off $<30 \mathrm{sec}$, grasped firmly with both front paws; 2 , did not fall $<30 \mathrm{sec}$, attempted to climb the wire; 3 , did not fall $<30 \mathrm{sec}$, held wire with both front paws and one or both hind paws; 4 , did not fall $<30 \mathrm{sec}$, clutched wire with all four limbs, wrapped tail around the wire and 5, did not fall $<30 \mathrm{sec}$, climbed to the end of the wire. Rats were scored in a blinded manner.

Brain water content. Brain water content was measured as described previously (25). The brain water content in each group was measured by the dry-wet weight method to evaluate brain edema following ICH. Brain water content was calculated as follows: (Wet weight - dry weight)/wet weight $\mathrm{x} 100 \%$.

$B B B$ permeability. Evans blue (EB) is a commonly used dye indicator in the laboratory. EB content in brain tissue is directly proportional to the degree of $\mathrm{BBB}$ damage. Therefore, EB is often used as a tracer to evaluate BBB integrity (26). At 3 days post-ICH, rats were intravenously injected with $2 \% \mathrm{~EB}$. Then, $3 \mathrm{~h}$ later, extravasated EB in the brain was 
A

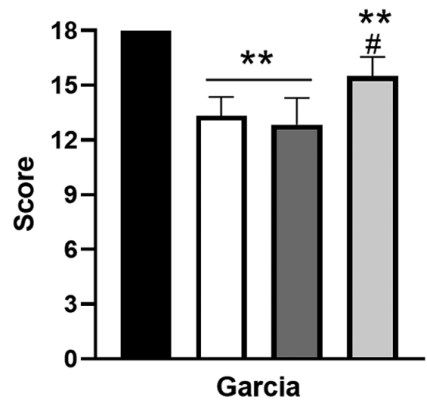

B

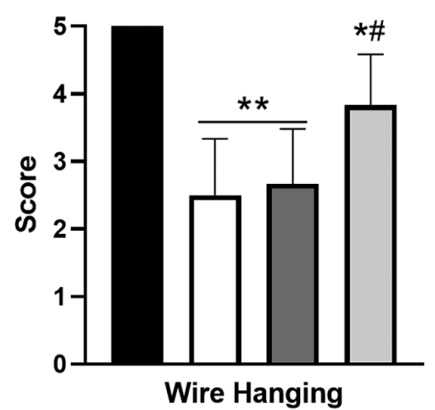

C

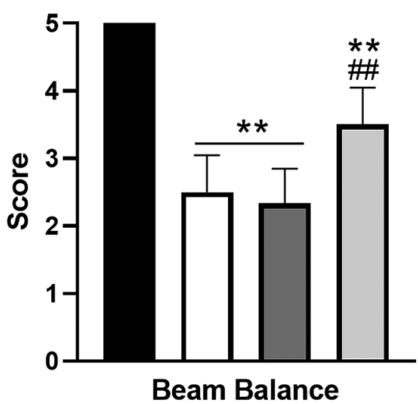

- Sham $\square \mathrm{ICH} \quad \square \mathrm{ICH}+$ Lenti.Null $\quad \square \mathrm{ICH}+$ Lenti.CTRP3

Figure 1. Effect of CTRP3 treatment on neurological function3 days after ICH. All animals following ICH showed significant neurological deficit based on performance on (A) modified Garcia, (B) wire hanging and (C) beam balance tests. Rats treated with Lenti-CTRP3 2 weeks before ICH showed less neurological deficit in all three tests. Data are presented as the mean \pm SEM. $n=9 /$ group. ${ }^{*} \mathrm{P}<0.05,{ }^{* *} \mathrm{P}<0.01$ vs. Sham; ${ }^{\# P}<0.05$, ${ }^{\# \#} \mathrm{P}<0.01$ vs. Lenti.Null. CTRP3, C1q/tumor necrosis factor-related protein-3; Lenti.CTRP3, lentivirus overexpressing CTRP3; ICH, intracerebral hemorrhage; Lenti.Null, lentivirus harboring empty vector.

evaluated by spectrophotometry (Thermo Fisher Scientific, Inc.) at $620 \mathrm{~nm}$.

Western blotting. Total protein from the perihematoma area of the rat striatum was extracted using ice-cold RIPA lysis buffer (Beyotime Institute of Biotechnology) supplemented with proteinase and phosphatase inhibitors. Protein concentration was determined by BCA assay. Then, a total of $50 \mu \mathrm{g}$ protein/lane was separated by $10 \%$ SDS-PAGE and transferred onto PVDF membranes. The membranes were blocked for $1.5 \mathrm{~h}$ at room temperature in 5\% non-fat-milk TBST (0.1\% Tween-20) buffer and incubated overnight at $4^{\circ} \mathrm{C}$ with a primary rabbit anti-rat CTRP3 (1:500; ab36870, Abcam,), SIRT1 (1:500; cat. no. BS64501, Bioworld Technology, Inc.), TNF- $\alpha$ (1:500; cat. no. BS6000, Bioworld Technology, Inc.), IL-1 $\beta$ (1:500; cat. no. BS6067, Bioworld Technology, Inc.) and $\beta$-actin (1:3,000; cat. no. AP0060, Bioworld Technology, Inc.) antibody. The membrane was incubated with secondary anti-rabbit antibody (1:4,000; horseradish peroxidase-conjugated Goat Anti-Rabbit IgG, cat. no. D110058, Sangon Biotech Co. Ltd.) for $1.5 \mathrm{~h}$ at room temperature. Images were captured using MicroChemi (Bio-Rad Laboratories, Inc.) and analyzed using ImageJ version 1.8.0 (National Institutes of Health) software.

ELISA. At 3 days following ICH, rats were deeply anesthetized and the perihematomal brain tissue homogenate was harvested. The tissue was homogenized with the BioVision fractionation kit (K256-100), centrifuged (1500 g, $\left.4^{\circ} \mathrm{C}, 30 \mathrm{~min}\right)$ and serum collected from the supernatant. The levels of TNF- $\alpha$ and IL-1 $\beta$ in peripheral blood were determined with ELISA kits (cat. nos. EK0526 and EK0394, respectively; Boster Biological Technology), according to the manufacturer's instructions.

Statistical analysis. All data are presented as the mean \pm SEM. One-way ANOVA followed by Tukey's post hoc test was used to compare results among all groups. SPSS 19.0 (IBM Corp.) software was used to perform all statistical analysis. $\mathrm{P}<0.05$ was considered to indicate a statistically significant difference.

\section{Results}

CTRP3 protects the brain from SBI in ICH rats. Modified Garcia, wire hang and beam balance test were used to investigate the effect of CTRP3 on ICH-induced neurological deficit. In the three tests, CTRP3 overexpression mitigated neurological deficit at 3 days after the induction of ICH (Fig. 1A-C). Brain edema volume was assessed by measuring brain water content. The brain water content of the ipsilateral side, particularly in the striatum, was significantly lower in the Lenti.CTRP3 group than in the Lenti.Null group (Fig. 2A). BBB permeability was assessed via EB extravasation in ICH rats. Significant accumulation of EB was seen in the ipsilateral hemispheres of ICH rats compared with that in sham rats (Fig. 2B). Treatment with CTRP3 significantly decreased extravasation in the ipsilateral hemisphere compared with the Lenti.Null group at 3 days after ICH (Fig. 2B). These results suggested that CTRP3 provided neuroprotection and attenuated SBI following ICH.

CTRP3 suppresses inflammatory responses in ICH rats. To investigate the function of CTRP3 in inflammatory responses in $\mathrm{ICH}$ rats, levels of inflammatory-associated cytokines were assessed in perihematomal brain tissue at 3 days after $\mathrm{ICH}$ via western blotting. Expression levels of proinflammatory cytokines TNF- $\alpha$ and IL- $1 \beta$ were significantly higher in the ICH group than in the sham group (Fig. 3). In addition, the Lenti.CTRP3 group exhibited significantly decreased expression levels of these factors compared with those in the Lenti.Null group (Fig. 3). ELISA revealed that the activity of TNF- $\alpha$ and IL-1 $\beta$ (Fig. 4A and B) was also decreased in the Lenti.CTRP3 group compared with the Lenti.Null group. Taken together, these findings suggested that CTRP3 protected against inflammation following $\mathrm{ICH}$.

CTRP3 activates the SIRT1 signaling pathway in ICH rats. To investigate the effect of CTRP3 in an ICH model, CTRP3 was successfully overexpressed in rats (Fig. 5A). To determine the mechanisms responsible for CTRP3-mediated neuroprotection at the molecular level, SIRT1 protein expression was 
A

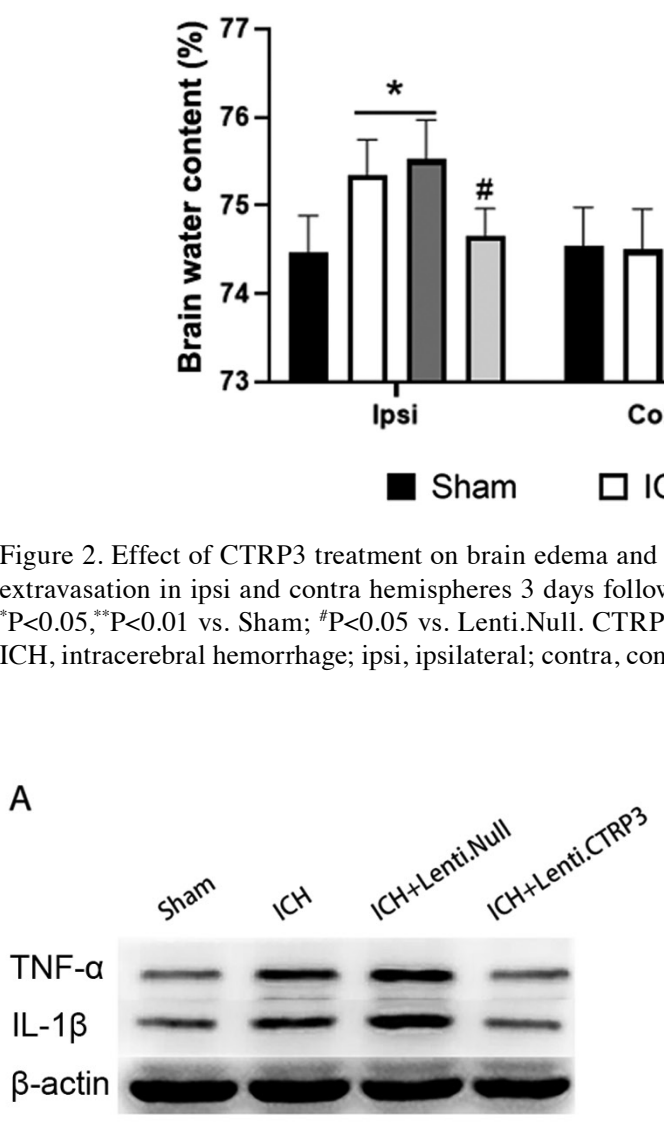

B

A
B

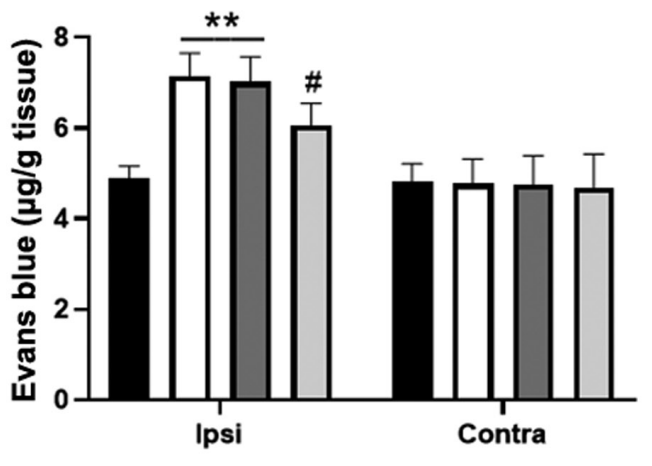

Figure 2. Effect of CTRP3 treatment on brain edema and BBB damage 3 days after ICH. (A) Brain water content and (B) quantification of Evans blue dye extravasation in ipsi and contra hemispheres 3 days following $\mathrm{ICH}$ in rats treated with Lenti-CTRP3. Data are presented as the mean \pm SEM. $\mathrm{n}=8 / \mathrm{group}$. ${ }^{*} \mathrm{P}<0.05,{ }^{* *} \mathrm{P}<0.01$ vs. Sham; ${ }^{\text {P }}<0.05$ vs. Lenti.Null. CTRP3, C1q/tumor necrosis factor-related protein-3; Lenti.CTRP3, lentivirus overexpressing CTRP3; $\mathrm{ICH}$, intracerebral hemorrhage; ipsi, ipsilateral; contra, contralateral; Lenti.Null, lentivirus harboring empty vector.
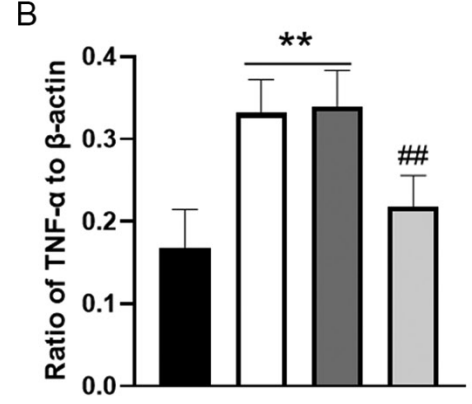

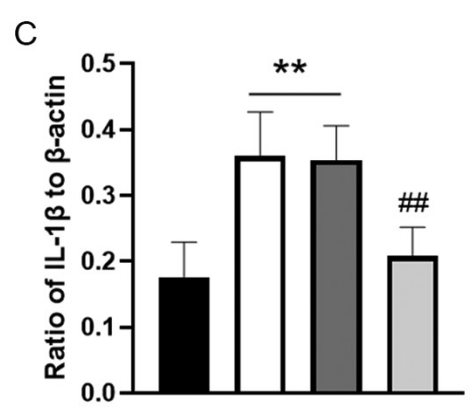

\section{口 Sham $\square \mathrm{ICH} \quad \square \mathrm{ICH}+$ Lenti.Null $\quad \square \mathrm{ICH}+$ Lenti.CTRP3}

Figure 3. Expression of TNF- $\alpha$ and IL-1 $\beta$ in the striatum 3 days after ICH. (A) Western blot analysis of TNF- $\alpha$ and IL-1 $\beta$. Quantified ratios of (B) TNF- $\alpha$ and (C) IL-1 $\beta$ to $\beta$-actin. Data are presented as mean \pm SEM. $n=7 /$ group. ${ }^{* *} \mathrm{P}<0.01$ vs. Sham; ${ }^{\# \#} \mathrm{P}<0.01$ vs. Lenti.Null. CTRP3, Clq/tumor necrosis factor-related protein-3; Lenti-CTRP3, lentivirus overexpressing CTRP3; ICH, intracerebral hemorrhage; Lenti.Null, lentivirus harboring empty vector.

A

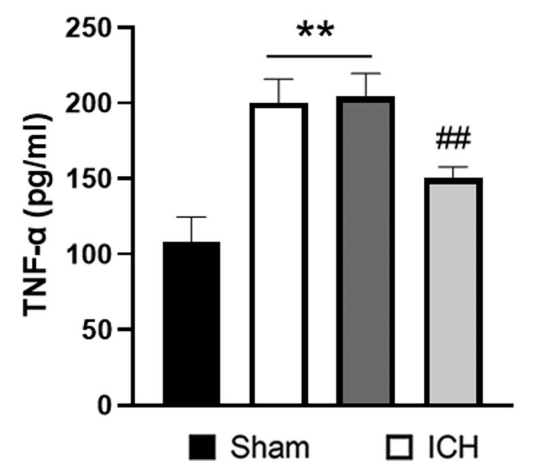

B

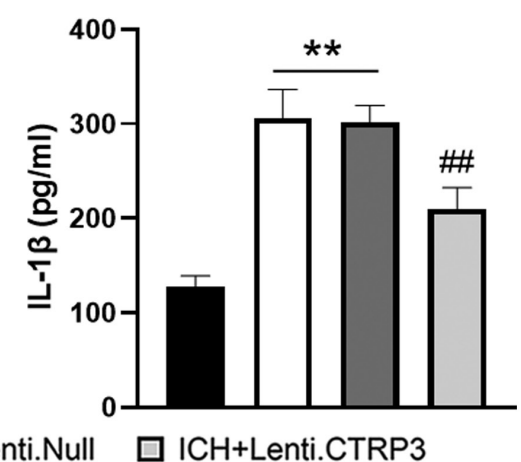

Figure 4. Effect of CTRP3 on expression of TNF- $\alpha$ and IL-1 $\beta$ in peripheral blood 3 days after ICH. (A) TNF- $\alpha$ and (B) IL-1 $\beta$ were examined via ELISA assay. Data are presented as the mean \pm SEM. $n=6$ /group. ${ }^{* *} \mathrm{P}<0.01$ vs. Sham; ${ }^{\# \#} \mathrm{P}<0.01$ vs. Lenti.Null. CTRP3, C1q/tumor necrosis factor-related protein-3; Lenti-CTRP3, lentivirus overexpressing CTRP3; ICH, intracerebral hemorrhage; Lenti.Null, lentivirus harboring empty vector.

measured in perihematomal brain tissue 3 days after ICH. Compared with the sham group, SIRT1 was increased in the ICH group and CTRP3 overexpression induced a further increase in expression of SIRT1 compared with that in Lenti. Null group at 3 days post-ICH (Fig. 5B and C).
Neuroprotective effects of CTRP3 are blocked by SIRT1 inhibition. Activation of SIRT1 occurred following CTRP3 overexpression (Fig. 6A-C) and inhibition of SIRT1 with EX527 abolished CTRP3-induced SIRT1 upregulation (Fig. 6A-C). Next, the effect of the SIRT1 signal 
A

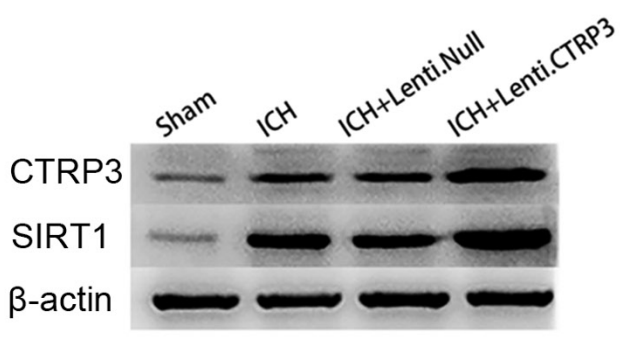

Sham
B

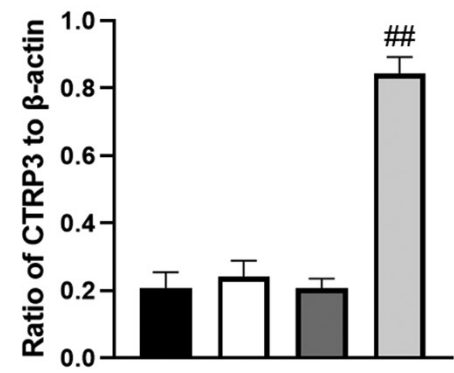

C

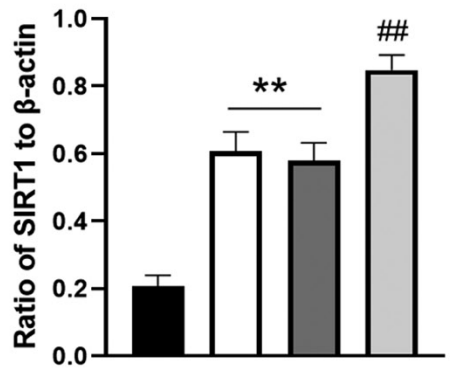

Figure 5. Effect of CTRP3 on expression of SIRT1 in the striatum 3 days following ICH. (A) Western blot analysis of the effect of Lenti-CTRP3 on CTRP3 and SIRT1 expression. Ratio of (B) CTRP3 and (C) SIRT1 protein expression. $n=5 /$ group. ${ }^{* *} \mathrm{P}<0.01$ vs. Sham; ${ }^{* \#} \mathrm{P}<0.01$ vs. Lenti.Null. CTRP3, C1q/tumor necrosis factor-related protein-3; Lenti-CTRP3, lentivirus overexpressing CTRP3; ICH, intracerebral hemorrhage; Lenti.Null, lentivirus harboring empty vector.

A

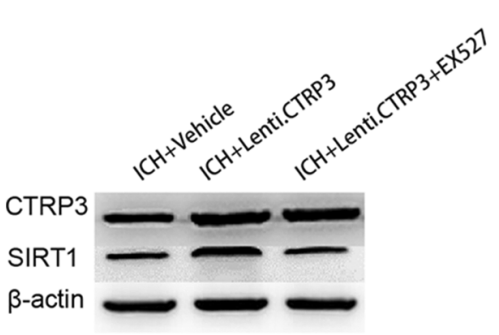

E

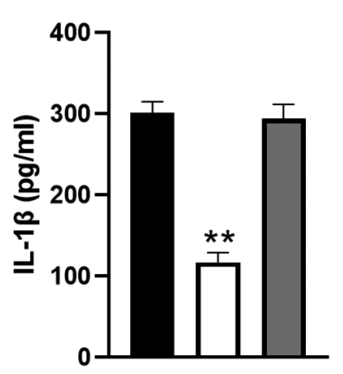

B

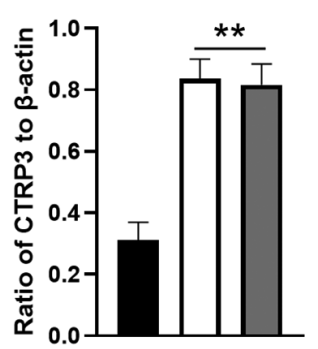

F

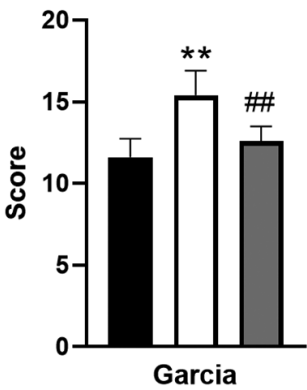

C

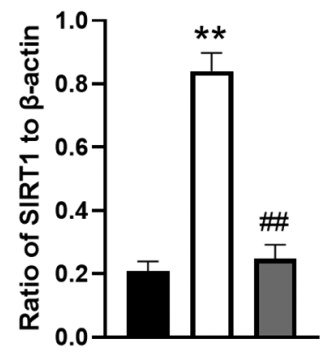

D

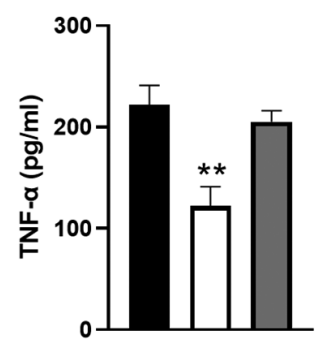

$\mathrm{ICH}+$ Vehicle

ICH+Lenti.CTRP3

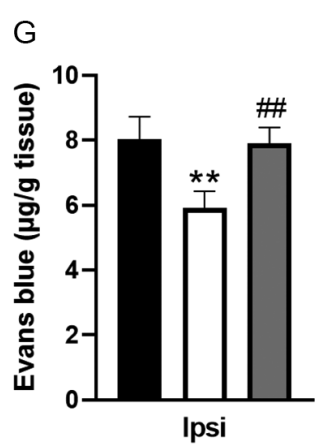

$\mathrm{H}$

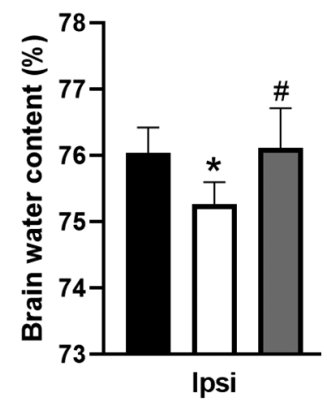

Figure 6. Neuroprotective effect of CTRP3 is blocked by SIRT1 inhibition. (A) Representative western blot bands of CTRP3 and SIRT1 in striatum of rats 3 days after ICH. Representative ratio of (B) CTRP3 and (C) SIRT1 to $\beta$-actin. (D) TNF- $\alpha$ and (E) IL-1 $\beta$ were assessed using ELISA assay. (F) Modified Garcia test was used to assess the effect of EX527 on neurological function improvement induced by Lenti.CTRP3. (G) Evans blue dye extravasation and $(\mathrm{H})$ brain water content were assessed to determine the effect of EX527 on ICH-induced BBB damage and brain edema decreased by Lenti.CTRP3 in Ipsi. Data are presented as the mean \pm SEM. $n=5 /$ group. ${ }^{*} \mathrm{P}<0.05,{ }^{* *} \mathrm{P}<0.01 \mathrm{vs}$. ICH + Vehicle; ${ }^{\#} \mathrm{P}<0.05,{ }^{\# \#} \mathrm{P}<0.01 \mathrm{vs}$. ICH + Lenti.CTRP3.CTRP3, C1q/tumor necrosis factor-related protein-3; Lenti-CTRP3, lentivirus overexpressing CTRP3; ICH, intracerebral hemorrhage; Lenti.Null, lentivirus harboring empty vector; ipsi, ipsilateral; SIRT1, silent information regulator 1; BBB, blood-brain barrier.

pathway in CTRP3-mediated suppression of ICH-induced inflammatory response was investigated. SIRT1-induced suppression of inflammatory responses was abrogated by EX527 treatment, which manifested as increased expression levels of TNF- $\alpha$ and IL-1 $\beta$ (Fig. 6D and E). Furthermore, EX527 blocked the neuroprotective effects of Lenti.CTRP3 (Fig. 6F-H). These results indicated that SIRT1 inhibition abolished the protective effects of CTRP3 in vivo.

\section{Discussion}

SBI is characterized as hematomal expansion caused by neuroinflammation that contributes to exacerbating functional outcomes following ICH $(27,28)$. The present study focused on CTRP3 as an approach to mitigate ICH-induced brain injury and dysfunction. The present results suggested that CTRP3 significantly alleviated neurobehavioral deficit, ICH-induced brain edema and breakdown of the BBB. Moreover, exogenous 
CTRP3 overexpression activated the SIRT1 signaling pathway, suggesting that the SIRT1 signaling pathway represents a potential mechanism by which CTRP3 protects against ICH. Furthermore, CTRP3 suppressed ICH-induced inflammatory responses in vivo. Collectively, the present findings provided a novel approach for the treatment of ICH-induced SBI.

Inflammation is one of the key processes underlying ICH-induced brain injury $(29,30)$. A previous study suggested that the mechanisms underlying brain edema formation are dependent on disruption of BBB integrity (31). Inflammation leads to increased vascular permeability and, ultimately, BBB damage (32-34). In the present study, CTRP3 overexpression resulted in maintenance of $\mathrm{BBB}$ integrity following ICH. ICH activates NF- $\kappa \mathrm{B}$, thus promoting release of inflammatory cytokines and ultimately leading to neurological dysfunction $(34,35)$. TNF- $\alpha$ and IL- $1 \beta$ are the most studied cytokines in ICH (36-38). Previous studies have shown that these two factors are increased significantly following ICH and are associated with brain edema $(39,40)$. The present study investigated whether CTRP3 inhibits ICH-induced neuroinflammation and found that CTRP3 decreased TNF- $\alpha$ and Il-1 $\beta$ levels. However, the pathway by which CTRP3 exerts its anti-inflammatory effects remains unclear. SIRT1 suppresses expression of NF- $\kappa \mathrm{B}$, which is the upstream regulator of TNF- $\alpha$ and IL-1 $\beta$ (41). In the present study, a SIRT1-specific inhibitor partially abolished CTRP3-mediated downregulation of TNF- $\alpha$ and IL- $1 \beta$ in ICH rats, implying a key role of SIRT1 as an anti-inflammatory effector of CTRP3.

SIRT1 is a member of the class III group of histone deacetylases and is activated in response to various types of cellular stressor, such as inflammation, cell death and metabolism. Accumulating evidence has indicated the role of SIRT1 in neurobehavioral deficit; activation of SIRT1 exhibits neuroprotective effects on cerebral ischemia or hemorrhagic stroke $(42,43)$. Zhou et al $(43)$ reported that SIRT1 protein levels are slightly increased following ICH. EX527 is a SIRT1 specific inhibitor; it significantly inhibits SIRT1 activity in vitro but has no effect on SIRT1 mRNA and protein expression. In vivo, EX527 not only inhibits the activity of SIRT1, but also downregulates expression of SIRT1 protein (44).Consistent with the aforementioned studies, here SIRT1 protein levels were increased following ICH in rats. Moreover, activating SIRT1 by CTRP3 in vivo attenuated $\mathrm{ICH}$-induced brain dysfunction and inhibition of SIRT1 abolished the protective effect provided by CTRP3 in ICH rats. These findings indicated that CTRP3-mediated amelioration of neurobehavioral deficit may be at least partly mediated by SIRT1.

As a novel adipokine, little is known about CTRP3-associated signaling pathways. The present study demonstrated that SIRT1 inhibition partially abolished CTRP3-mediated neuroprotection in ICH rats, implying that CTRP3 may suppress ICH-induced SBI via activating SIRT1. These findings reveal the complexity of signaling pathways regulated by CTRP3. Further studies are needed to advance understanding of the precise mechanisms by which CTRP3 exerts its biological effects.

In conclusion, the present study demonstrated that CTRP3 prevented the aggravation of brain edema and neuronal damage following ICH in vivo. CTRP3 inhibited inflammation via the SIRT1 pathway and may have protected the BBB, as well as neurons and glia, against SBI following ICH. Thus, the present findings suggested that CTRP3 may be an effective therapeutic candidate for the treatment of SBI following ICH.

\section{Acknowledgements}

Not applicable.

\section{Funding}

The present study was supported by grants from Natural Science Foundation of the Sichuan Provincial Education Office (grant no. 18ZA0529),Science Foundation of Southwest Medical University (grant no. 0903-00030982), The Science Foundation of the Affiliated Hospital of Southwest Medical University (grant no. 2017-PT-7) and The Doctoral Scientific Research Start-up Fund Project of the Affiliated Hospital of Southwest Medical University (grant no. 18058).

\section{Availability of data and materials}

The datasets used and/or analyzed during the current study are available from the corresponding author on reasonable request.

\section{Authors' contributions}

YW and SW confirm the authenticity of all the raw data. YW and SW designed the research and performed the analysis. JW assisted in study design. BY, CH, XT, HY and YL performed experiments and analyzed data. YW and SW drafted and revised the manuscript. All authors have read and approved the final manuscript.

\section{Ethics approval and consent to participate}

All experimental procedures were in accordance with the guidelines for the care and use of laboratory animals of the National Institutes of Health. All animal studies were approved by the Biomedical Ethics Committee of Southwest Medical University (approval no. 20210223-135).

\section{Patient consent for publication}

Not applicable.

\section{Competing interests}

The authors declare that they have no competing interests.

\section{References}

1. Gunda B, Böjti P and Kozák LR: Hyperacute Spontaneous Intracerebral Hemorrhage During Computed Tomography Scanning. JAMA Neurol 78: 365-366, 2021.

2. Cho S, Rehni AK and Dave KR: Tobacco Use: A Major Risk Factor of Intracerebral Hemorrhage. J Stroke 23: 37-50, 2021

3. Wu YC, Ding Z, Wu J, Wang YY, Zhang SC, Wen Y, Dong WY and Zhang QY: Increased glycemic variability associated with a poor 30-day functional outcome in acute intracerebral hemorrhage. J Neurosurg 129: 861-869, 2018. 
4. Kuramatsu JB, Biffi A, Gerner ST, Sembill JA, Sprügel MI, Leasure A, Sansing L, Matouk C, Falcone GJ, Endres M, et al: Association of surgical hematoma evacuation vs conservative treatment with functional outcome in patients with cerebellar intracerebral hemorrhage. JAMA 322: 1392-1403, 2019.

5. Sugiyama T, Imai T, Nakamura S, Yamauchi K, Sawada S, Shimazawa $M$ and Hara H: A novel Nrf2 activator, RS9, attenuates secondary brain injury after intracerebral hemorrhage in sub-acute phase. Brain Res 1701: 137-145, 2018.

6. Atangana E, Schneider UC, Blecharz K, Magrini S, Wagner J, Nieminen-Kelhä M, Kremenetskaia I, Heppner FL, Engelhardt B and Vajkoczy P: Intravascular Inflammation Triggers Intracerebral Activated Microglia and Contributes to Secondary Brain Injury After Experimental Subarachnoid Hemorrhage (eSAH). Trans Stroke Res 8: 144-156, 2017.

7. Wang Z, Zhou F, Dou Y, Tian X, Liu C, Li H, Shen H and Chen G: Melatonin Alleviates Intracerebral Hemorrhage-Induced Secondary Brain Injury in Rats via Suppressing Apoptosis, Inflammation, Oxidative Stress, DNA Damage, and Mitochondria Injury. Transl Stroke Res 9: 74-91, 2018.

8. Walsh KB, Zhang X, Zhu X, Wohleb E, Woo D, Lu L and Adeoye O: Intracerebral Hemorrhage Induces Inflammatory Gene Expression in Peripheral Blood: Global Transcriptional Profiling in Intracerebral Hemorrhage Patients. DNA Cell Biol 38: 660-669, 2019

9. Wang J and Doré S: Inflammation after intracerebral hemorrhage. J Cereb Blood Flow Metab 27: 894-908, 2007.

10. Tschoe C, Bushnell CD, Duncan PW, Alexander-Miller MA and Wolfe SQ: Neuroinflammation after Intracerebral Hemorrhage and Potential Therapeutic Targets. J Stroke 22: 29-46, 2020

11. Zhu H, Wang Z, Yu J, Yang X, He F, Liu Z, Che F, Chen X, Ren H, Hong M and Wang J. : Role and mechanisms of cytokines in the secondary brain injury after intracerebral hemorrhage. Prog Neurobiol 178: 101610, 2019.

12. Gao K, Niu J and Dang X: Neuroprotection of melatonin on spinal cord injury by activating autophagy and inhibiting apoptosis via SIRT1/AMPK signaling pathway. Biotechnol Lett 42: 2059-2069, 2020 .

13. Vellimana AK, Aum DJ, Diwan D, Clarke JV, Nelson JW, Lawrence M, Han BH, Gidday JM and Zipfel GJ: SIRT1 mediates hypoxic preconditioning induced attenuation of neurovascular dysfunction following subarachnoid hemorrhage. Exp Neurol 334: 113484, 2020.

14. Deng HJ, Zhou CH, Huang LT, Wen LB, Zhou ML and Wang CX: Activation of silent information regulator 1 exerts a neuroprotective effect after intracerebral hemorrhage by deacetylating

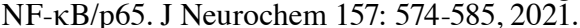

15. Ahima RS, Qi Y, Singhal NS, Jackson MB and SchererPE: Brain adipocytokine action and metabolic regulation. Diabetes 55 (Suppl 2): S145-S154, 2006.

16. Schmid A, Gehl J, Thomalla M, Hochberg A, Kreiss A, Patz M, Karrasch T and Schäffler A: Downregulation of CTRP-3 by Weight Loss In Vivo and by Bile Acids and Incretins in Adipocytes In Vitro. Int J Mol Sci 21: 8168, 2020

17. Liu Y, Li LN, Guo S, Zhao XY, Liu YZ, Liang C, Tu S, Wang D, Li L, Dong JZ, et al: Melatonin improves cardiac function in a mouse model of heart failure with preserved ejection fraction. Redox Biol 18: 211-221, 2018

18. Yuan YP, Ma ZG, Zhang X, Xu SC, Zeng XF, Yang Z, Deng W and Tang QZ: CTRP3 protected against doxorubicin-induced cardiac dysfunction, inflammation and cell death via activation of Sirt1. J Mol Cell Cardiol 114: 38-47, 2018.

19. National Research Council (US) Committee for the Update of the Guide for the Care and Use of Laboratory Animals: Guide for the care and use of laboratory animals: 8th edition. The National Academies Press, Washington, DC, 2011

20. Wang S, Zhou Y, Yang B, Li L, Yu S, Chen Y, Zhu J and Zhao Y: C1q/Tumor Necrosis Factor-Related Protein-3 Attenuates Brain Injury after Intracerebral Hemorrhage via AMPK-Dependent Pathway in Rat. Front Cell Neurosci 10: 237, 2016.

21. Berg T: Kv7(KCNQ)-K+-Channels Influence Total Peripheral Resistance in Female but Not Male Rats, and Hamper Catecholamine Release in Hypertensive Rats of Both Sexes. Front Physiol 9: 117, 2018.

22. Liu SP, Huang L, Flores J, Ding Y, Li P, Peng J, Zuo G, Zhang JH, Lu J and Tang JP: Secukinumab attenuates reactive astrogliosis via IL-17RA/(C/EBP $\beta) / S I R T 1$ pathway in a rat model of germinal matrix hemorrhage. CNS Neurosci Ther 25: $1151-1161,2019$
23. Yue X, Liu L, Yan H, Gui Y, Zhao J and Zhang P: Intracerebral Hemorrhage Induced Brain Injury Is Mediated by the Interleukin-12 Receptor in Rats. Neuropsychiatr Dis Treat 16 891-900, 2020

24. Wang S, Yao Q, Yu W, Wang JQ, Huang CG, Li D and Yang B: Adiponectin reduces brain injury after intracerebral hemorrhage by reducing NLRP3 inflammasome expression. Int J Neurosci 130: 301-308, 2020.

25. Xie L, Wang Y, Chen Z: EGR1 knockdown alleviates the cerebral injury in rats with intracerebral hemorrhage (ICH) via STAT3/NF- $\kappa \mathrm{B}$ pathway by reducing $\mathrm{RXR} \alpha$ acetylation level. Neuroscience: Feb 16, 2021 (Epub ahead of print).

26. Zhao H, Zhang K, Tang R, Meng H, Zou Y, Wu P, Hu R, Liu X, Feng $\mathrm{H}$ and Chen Y: Trpv4 blockade preserves the blood-brain barrier by inhibiting stress fiber formation in a rat model of intracerebral hemorrhage. Front Mol Neurosci 11: 97, 2018.

27. James ML, Komisarow JM, Wang $\mathrm{H}$ and Laskowitz DT: Therapeutic Development of Apolipoprotein E Mimetics for Acute Brain Injury: Augmenting Endogenous Responses to Reduce Secondary Injury. Neurotherapeutics 17: 475-483, 2020.

28. Mazzeo AT, Filippini C, Rosato R, Fanelli V, Assenzio B, Piper I, Howells T, Mastromauro I, Berardino M, Ducati A, et al: Multivariate projection method to investigate inflammation associated with secondary insults and outcome after human traumatic brain injury: A pilot study. J Neuroinflammation 13: 157-157, 2016.

29. Xiao H, Chen H, Jiang R, Zhang L, Wang L, Gan H, Jiang N, Zhao J, Zhai X and Liang P: NLRP6 contributes to inflammation and brain injury following intracerebral haemorrhage by activating autophagy. J Mol Med (Berl) 98: 1319-1331, 2020.

30. Li Z, He Q, Zhai X, You Y, Li L, Hou Y, He F, Zhao Y and Zhao J: Foxo1-mediated inflammatory response after cerebral hemorrhage in rats. Neurosci Lett 629: 131-136, 2016.

31. Zhu J, Li X, Yin J, Hu Y, Gu Y and Pan S: Glycocalyx degradation leads to blood-brain barrier dysfunction and brain edema after asphyxia cardiac arrest in rats. J Cereb Blood Flow Metab 38: 1979-1992, 2018

32. Zhao F, Deng J, Xu X, Cao F, Lu K, Li D, Cheng X, Wang X and Zhao Y: Aquaporin-4 deletion ameliorates hypoglycemia-induced BBB permeability by inhibiting inflammatory responses. J Neuroinflammation 15: 157, 2018.

33. Varatharaj A and Galea I: The blood-brain barrier in systemic inflammation. Brain Behav Immun 60: 1-12, 2017.

34. Zhou F, Jiang Z, Yang B and Hu Z: Magnolol exhibits anti-inflammatory and neuroprotective effects in a rat model of intracerebral haemorrhage. Brain Behav Immun 77: 161-167, 2019.

35. Wu CH, Chen CC, Lai CY, Hung TH, Lin CC, Chao M and Chen SF: Treatment with TO901317, a synthetic liver $\mathrm{X}$ receptor agonist, reduces brain damage and attenuates neuroinflammation in experimental intracerebral hemorrhage. J Neuroinflammation 13: 62, 2016.

36. Shang Y, Dai S, Chen X, Wen W and Liu X: MicroRNA-93 regulates the neurological function, cerebral edema and neuronal apoptosis of rats with intracerebral hemorrhage through TLR4/NF- $\kappa \mathrm{B}$ signaling pathway. Cell Cycle 18: 3160-3176, 2019.

37. Chen S, Peng J, Sherchan P, Ma Y, Xiang S, Yan F, Zhao H, Jiang Y, Wang N, Zhang JH, et al: TREM2 activation attenuates neuroinflammation and neuronal apoptosis via PI3K/Akt pathway after intracerebral hemorrhage in mice. J Neuroinflammation 17: $168,2020$.

38. Shi SX, Li YJ, Shi K, Wood K, Ducruet AF and Liu Q: IL (Interleukin)-15 Bridges Astrocyte-Microglia Crosstalk and Exacerbates Brain Injury Following Intracerebral Hemorrhage. Stroke 51: 967-974, 2020

39. Chen S, Zhao L, Sherchan P, Ding Y, Yu J, Nowrangi D, Tang J, $\mathrm{Xia} \mathrm{Y}$ and Zhang JH: Activation of melanocortin receptor 4 with RO27-3225 attenuates neuroinflammation through AMPK/JNK/p38 MAPK pathway after intracerebral hemorrhage in mice. J Neuroinflammation 15: 106, 2018.

40. Gong Y, Wu M, Shen J, Tang J, Li J, Xu J, Dang B and Chen G: Inhibition of the NKCC1/NF- $\mathrm{B}$ Signaling Pathway Decreases Inflammation and Improves Brain Edema and Nerve Cell Apoptosis in an SBI Rat Model. Front Mol Neurosci 14: 641993, 2021. 
41. Han S, Li Z, Han F, Jia Y, Qi L, Wu G, Cai W, Xu Y, Li C, Zhang $\mathrm{W}$ and $\mathrm{Hu} \mathrm{D}$ : ROR alpha protects against LPS-induced inflammation by down-regulating SIRT1/NF-kappaB pathway, Arch Biochem Biophy 668: 1-8, 2019.

42. Hu Q, Manaenko A, Bian H, Guo Z, Huang JL, Guo ZN, Yang P, Tang $\mathrm{J}$ and Zhang JH: Hyperbaric Oxygen Reduces Infarction Volume and Hemorrhagic Transformation Through ATP/NAD+/Sirt1 Pathway in Hyperglycemic Middle Cerebral Artery Occlusion Rats. Stroke 48: 1655-1664, 2017.

43. Zhou Y, Wang S, Li Y, Yu S and Zhao Y: SIRT1/PGC-1a Signaling Promotes Mitochondrial Functional Recovery and Reduces Apoptosis after Intracerebral Hemorrhage in Rats. Front Mol Neurosci 10: 443, 2018
44. Shi YH, Zhang XL, Ying PJ, Wu ZQ, Lin LL, Chen W, Zheng GQ and Zhu WZ: Neuroprotective Effect of Astragaloside IV on Cerebral Ischemia/Reperfusion Injury Rats Through Sirt1/Mapt Pathway. Front Pharmacol 12: 639898, 2021.

cc) (i) $\odot$ This work is licensed under a Creative Commons Attribution-NonCommercial-NoDerivatives 4.0 International (CC BY-NC-ND 4.0) License. 